\title{
Communicating Hydrocephalus Accompanied by Arachnoid Cyst in Aneurismal Subarachnoid Hemorrhage
}

\author{
Jae Young Choi, Seung Heon Cha, Won Ho Cho, Jun Kyeung Ko \\ Department of Neurosurgery, Medical Research Institute, Pusan National University Hospital, Busan, Korea
}

The authors describe a case of communicating hydrocephalus accompanied by an arachnoid cyst in an aneurismal subarachnoid hemorrhage. A 69-year-old female was referred to our clinic due to the sudden onset of a headache. A head computed tomography scan demonstrated an arachnoid cyst in the right middle fossa with a mass effect and diffuse subarachnoid hemorrhage. Digital subtraction angiography then revealed a left internal carotid-posterior communicating artery aneurysm. The neck of the aneurysm was clipped successfully and the post-operative period was uneventful. However, two months after discharge, the patient reported that her mental status had declined over previous weeks. A cranial computed tomography scan revealed an interval increase in the size of the ventricle and arachnoid cyst causing a midline shift. Simultaneous navigation guided ventriculoperitoneal shunt and cystoperitoneal shunt placement resulted in remarkable radiological and clinical improvements.

Keywords Arachnoid cyst, Cerebral aneurysm, Hydrocephalus, Shunt, Subarachnoid hemorrhage
J Cerebrovasc Endovasc Neurosurg.

2013 December; 15(4):311-315

Received : 16 August 2013

Revised : 17 October 2013

Accepted : 31 October 2013

Correspondence to Jun Kyeung Ko

Department of Neurosurgery, Pusan National University Hospital, 305 Gudeok-ro, Seo-gu, Busan 602-739, Korea
Tel : 82-51-240-7937
Fax : 82-51-244-0282
E-mail : redcheek09@naver.com

This work was supported by a clinical research grant from Pusan National University Hospital 2012.

\section{INTRODUCTION}

Several reports on intracranial arachnoid cysts (ACs) have placed special emphasis on their association with hydrocephalus, and in the majority of these reports an effort was made to explain the linkage between hydrocephalus development and ACs. ${ }^{5}$ (11)12) However, somewhat surprisingly, little is known about the association between a preexisting $\mathrm{AC}$ and acquired hydrocephalus in aneurismal subarachnoid hemorrhage (SAH). Furthermore, to the best of our knowledge, no case of communicating hydrocephalus accompanied by AC in aneurismal SAH has been reported. We report a unique case of communicating hydrocephalus accompanied by an AC in aneurismal SAH.

\section{CASE REPORT}

A 69-year-old female with no significant prior medical history was referred to our clinic due to a headache of sudden onset. Head computed tomography (CT) scan performed at the time demonstrated an AC in the right middle fossa with a mass effect and diffuse SAH (Fig. 1). On clinical examination, the patient appeared lethargic and drowsy with a Glasgow coma scale score of 13 (E3, M6, V4), a Hunt-Hess grade of III, and a Fisher grade of IV.

Left carotid angiography revealed a left internal carotid-posterior communicating artery aneurysm, with a postero-laterally projecting dome (Fig. 2). Craniotomy was performed and complete aneurysm neck clipping and posterior communicating artery patency were confirmed intraoperatively by ultrasound using a mi- 


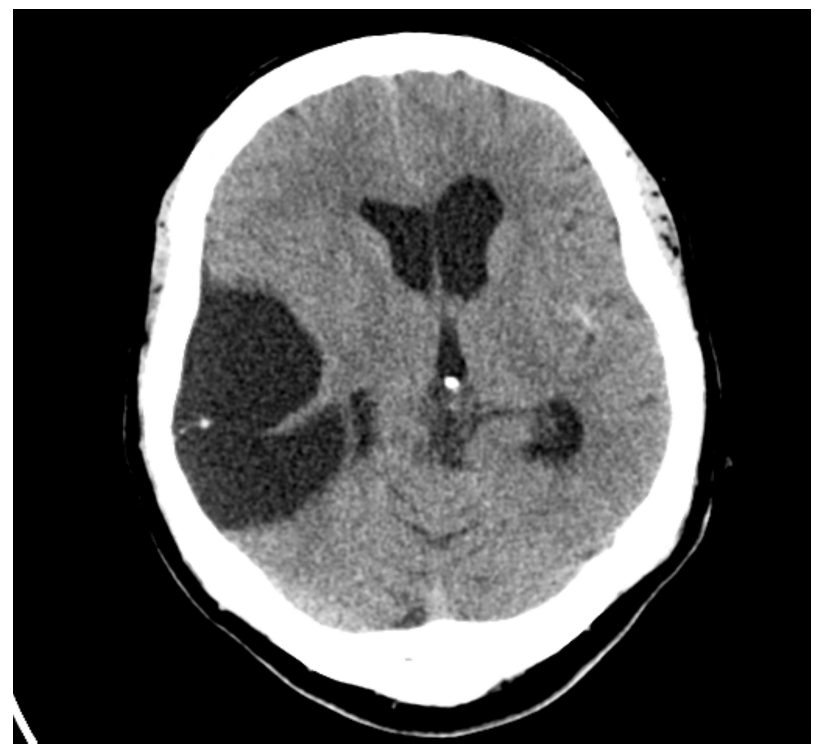

Fig. 1. The initial computed tomography (CT) scan shows a large, hypodense lesion within the right middle cranial fossa, causing a midline shift with right lateral ventricle compression, and scanty subarachnoid hemorrhage in the left sylvian fissure.

cro-Doppler probe (Fig. 3). The post-operative period was uneventful. The patient's consciousness level gradually improved, and she was discharged on the 17th post-operative day. However, at two months after discharge, she reported that her mental status had been declining over recent weeks. A neurological examination performed at the time revealed decreased consciousness, that is, severe confusion with gait dis-

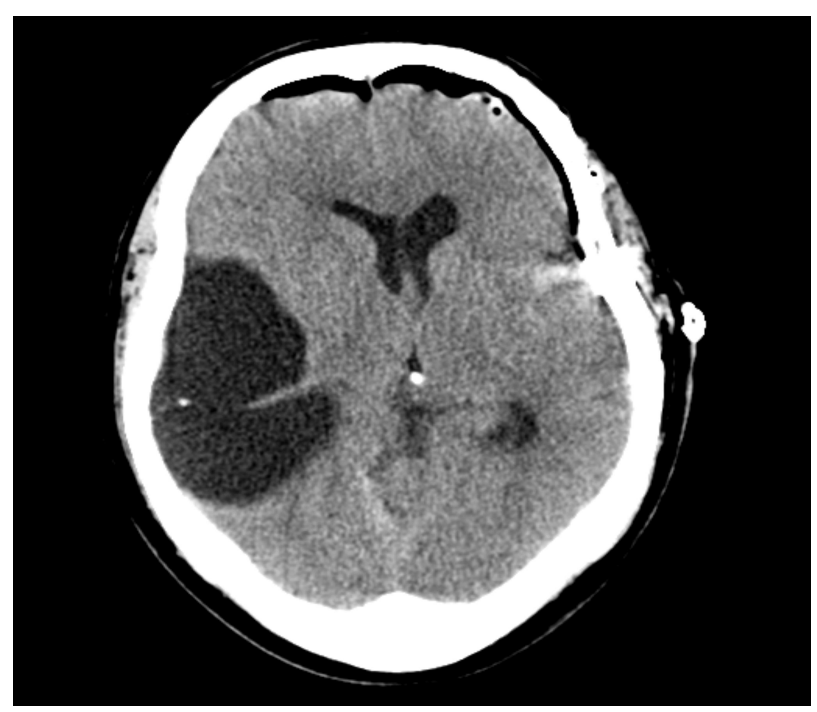

Fig. 3. Postoperative CT scan showing decreased ventricle size.

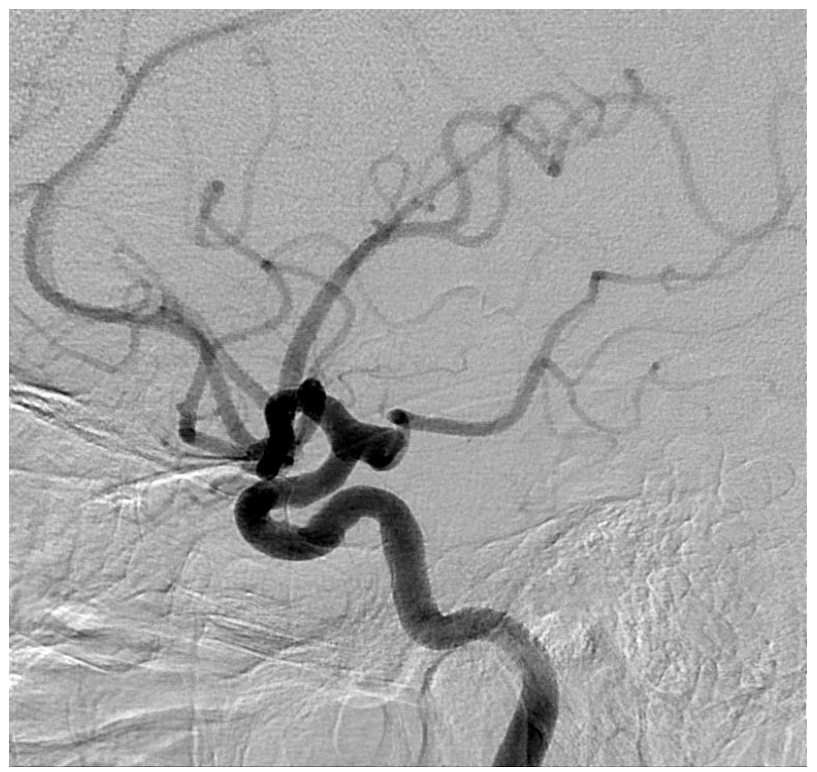

Fig. 2. Left internal carotid angiogram depicting a left internal carotid-posterior communicating artery aneurysm projecting posteriorly and laterally.

turbance and urinary incontinence. Cranial CT revealed an interval increase in the size of the ventricle and an AC causing a midline shift (Fig. 4). We planned simultaneous ventriculoperitoneal shunt and cystoperitoneal shunt placement to correct the enlarged ventricle and AC. The patient was positioned supine with her head turned left to expose the right temporal scalp. A curvilinear skin incision was made over the

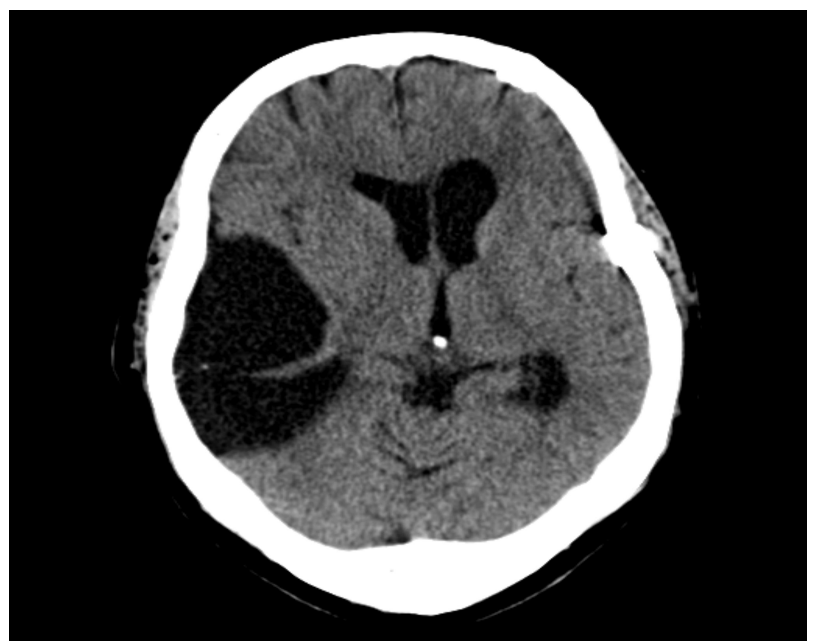

Fig. 4. CT scan obtained two months after operation showing an interval increase in ventricle and arachnoid cyst sizes causing a midline shift. 
temporo-occipital scalp for cyst access and another linear incision was made on the right Kocher's point for ventricular access by an intraoperative neuro-navigation system. Two intracranial catheters were connected using a Y-shaped titanium connector at the gate of the single valve (Fig. 5). A linear skin incision was made in the right upper abdomen to access the peritoneum for the distal end of the shunt catheter, and a programmable valve and distal catheter were tunnelled and passed in the usual manner.

The neurological deficits gradually subsided after the procedure and, two weeks later, the patient was discharged to home with no neurological deficit. Follow-up CT performed at one year post-operatively showed proper positioning of the shunt components and an interval improvement in brain condition including the hydrocephalus (Fig. 6).

\section{DISCUSSION}

Intracranial aneurysms and ACs are relatively common cerebral malformations, but coincidental occurrence between these two disorders are rare. ${ }^{314)}$ Including our

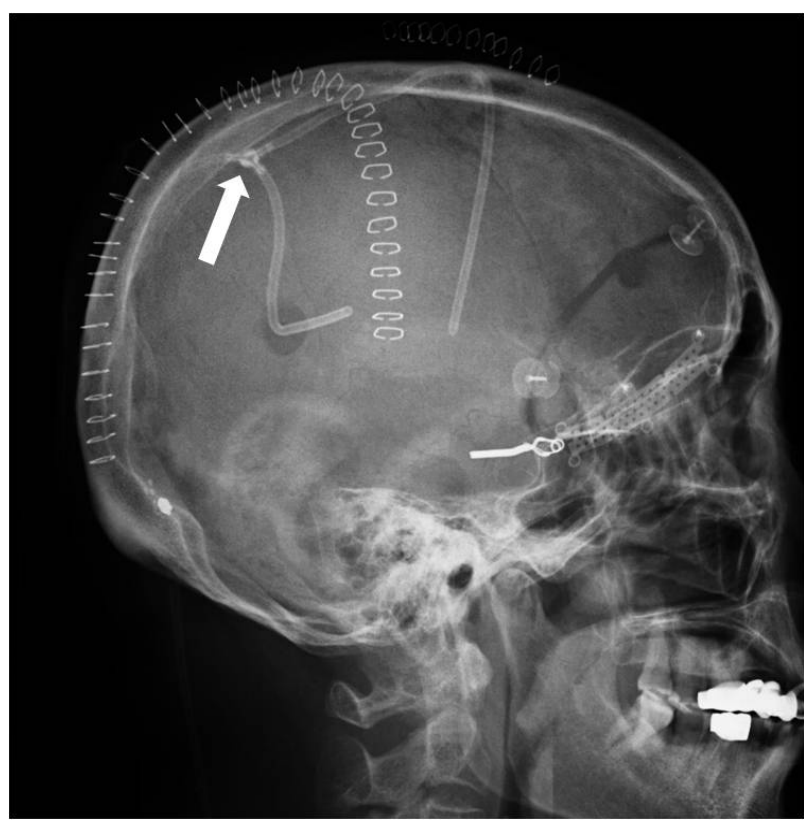

Fig. 5. Lateral skull radiograph demonstrating 2 intracranial catheters in the cyst and lateral ventricle connected by a titanium Y-shaped connector (arrow) at the gate of the single valve.

new case, only ten cases have been published. ${ }^{12)(6-8)(10) 13) 14)}$ Hirose et al. $\left.{ }^{6}\right)$ were the first to reported a case of intracystic hematoma without SAH due to a ruptured aneurysm. Similarly, Kajiwara et al. ${ }^{9)}$ described a case
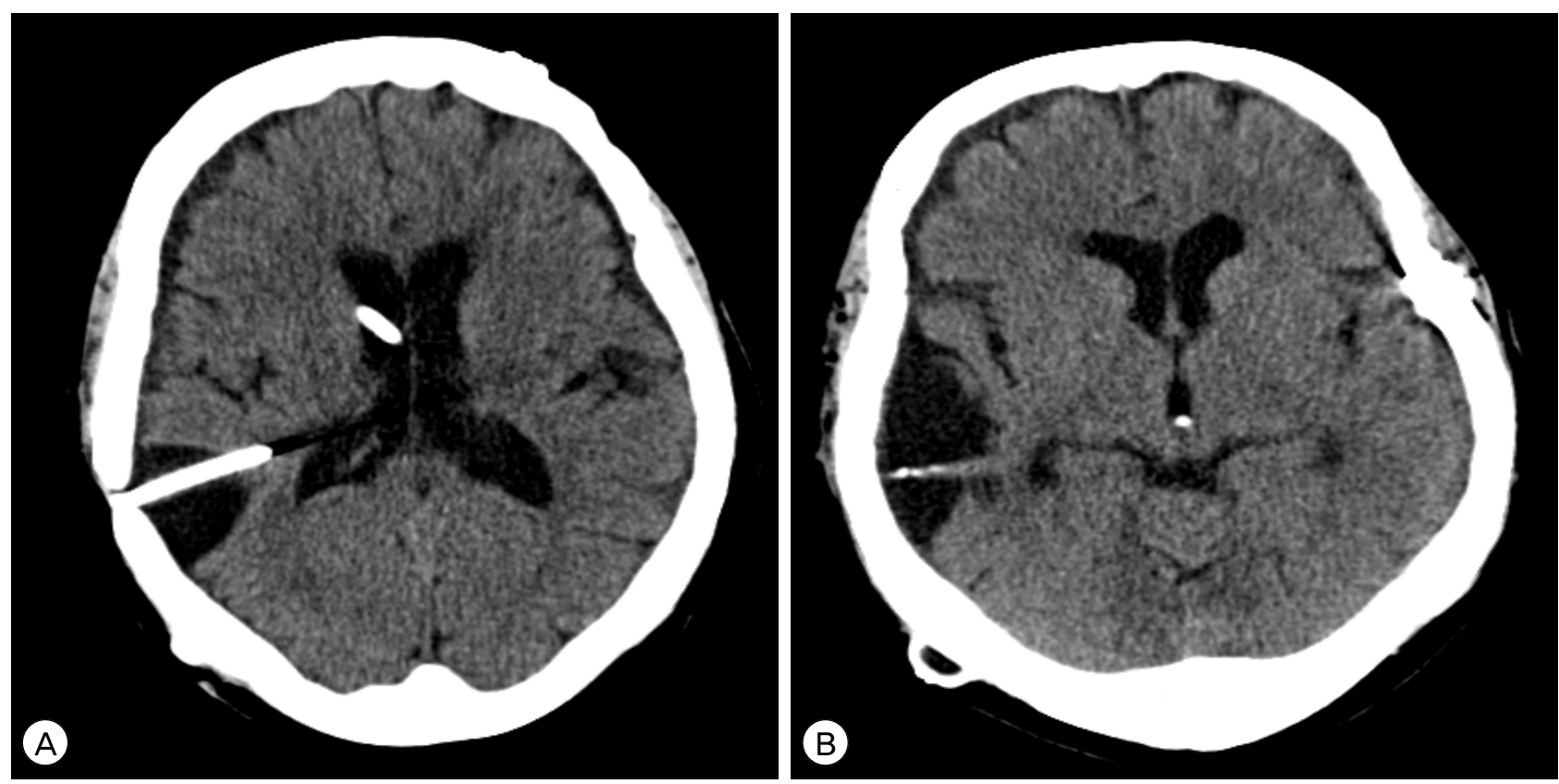

Fig. 6. Follow-up CT scans obtained at about one year post-operatively show proper positioning of shunt components (A) and an interval decrease in ventricles and cyst sizes (B). 
of $\mathrm{AC}$ in the middle fossa with intracystic hematoma caused by the rupture of an aneurysm arising from the internal carotid-posterior communicating artery. Furthermore, it was recommended that the coexistence of $\mathrm{AC}$ and a ruptured aneurysm be considered, indicating the need for cerebral angiography to investigate an intracystic hematoma of an $\mathrm{AC}$, especially in the absence of head injury. However, although most authors have described the characteristics of hemorrhage due to aneurismal rupture in a case of coexistent $\mathrm{AC}$, no mention has been made of the effect of a preexisting AC with hydrocephalus occurring after aneurismal SAH or their interactions.

Depending on the size of the cystic opening and on cerebrospinal fluid (CSF) dynamics, ACs may be classified as communicating or non-communicating according to their relationships with subarachnoid spaces. ACs may expand by the following mechanisms: (a) by a ball-valve action that allows the entry of CSF into a cyst but prevents egress, (b) by the intracystic production of fluid by secreting cells, occasionally found in the cyst walls, or (c) by movements of fluids driven by osmotic gradients. ${ }^{11)}$

In the present case, we believe that the middle fossa AC communicated with subarachnoid space and was asymptomatic before the aneurismal $\mathrm{SAH}$, but that delayed hydrocephalus resulting from the aneurismal SAH inflated the middle fossa AC gradually and caused a midline shift. We recall that her mental status at the time of the shunt operation was worse than usually encountered in patients suffering from delayed hydrocephalus after aneurismal SAH. Therefore, we suggest that a preexisting communicating AC can exacerbate the symptoms of hydrocephalus after SAH because of the mass effect of the expanded AC.

With regards to treating hydrocephalus in this case, at first we considered a ventriculoperitoneal shunt or cystoperitoneal shunt independently because we believed that there was communication between the cyst and subarachnoid space. However, some authors suggest that preceding or coexistent hydrocephalus is important in AC expansion, ${ }^{412)}$ explaining the develop- ment of ventriculomegaly or hydrocephalus following cyst shunting or, on the contrary, AC growth following the extracranial drainage of ventricular $\mathrm{CSF}^{12)}$ However, we were concerned about unpredictable changes in CSF dynamics after single shunt placement, and thus, finally decided on simultaneous ventriculoperitoneal shunt and cystoperitoneal shunt placement to treat the enlarged ventricle and $\mathrm{AC}$. Fortunately, the result of surgery was highly satisfactory both clinically and radiologically.

\section{CONCLUSION}

We document an uncommon case of communicating hydrocephalus accompanied by AC in aneurismal $\mathrm{SAH}$, describe its clinical features, and include a review of the literature. This case demonstrates that a preexisting communicating $\mathrm{AC}$ can exacerbate the symptom of hydrocephalus occurring after aneurismal SAH because of the mass effect of the expanded AC.

\section{REFERENCES}

1. Barker RA, Phillips RR, Moseley IF, Taylor WJ, Kitchen ND, Scadding JW. Posterior communicating artery aneurysm presenting with haemorrhage into an arachnoid cyst. J Neurol Neurosurg Psychiatry. 1998 Apr;64(4):558-60.

2. Baykal S, Ceylan S, Dinc H, Soylev E, Usul H, Akturk F. Aneurysm of an azygos anterior cerebral artery: Report of two cases and review of the literature. Neurosurg Rev. 1996 Jan;19(1):57-9.

3. de Oliveira JG, Giudicissi-Filho M, Rassi-Neto A, Borba LA, Rassi MS, Sanchez SL Jr, et al. Intracranial aneurysm and arachnoid cyst: A rare association between two cerebral malformations. Br J Neurosurg. 2007 Aug;21(4):406-10.

4. di Rocco C, Caldarelli M, di Trapani G. Infratentorial arachnoid cysts in children. Childs Brain. 1981 Jan;8(2):119-33.

5. Galassi E, Piazza G, Gaist G, Frank F. Arachnoid cysts of the middle cranial fossa: A clinical and radiological study of 25 cases treated surgically. Surg Neurol. 1980 Sep;14(3):211-9.

6. Hirose S, Shimada S, Yamaguchi N, Hosotani K, Kawano $\mathrm{H}$, Kubota T. Ruptured aneurysm associated with arachnoid cyst: Intracystic hematoma without subarachnoid hemorrhage. Surg Neurol. 1995 Apr;43(4):353-6.

7. Huang D, Abe T, Kojima K, Tanaka N, Watauabe M, Ohkura A, et al. Intracystic hemorrhage of the middle fossa arachnoid cyst and subdural hematoma caused by ruptured middle cerebral artery aneurysm. AJNR Am J 
Neuroradiol. 1999 Aug;20(7):1284-6.

8. Jinkins JR, Siqueira EB, Holoubi A. Ruptured middle cerebral aneurysm with accumulation of subarachnoid blood within convexity arachnoid cyst. Comput Radiol. 1987 Jul-Aug;11(4):185-7.

9. Kajiwara I, Tanaka T, Kan I, Ohtsuka T, Sawauchi S, Murakami S, et al. Intracystic hematoma of middle fossa arachnoid cyst caused by rupture of internal carotid-posterior communicating artery aneurysm. Neurol Med Chir (Tokyo). 2008 May;48(5):220-2.

10. Leo JS, Pinto RS, Hulvat GF, Epstein F, Kricheff II. Computed tomography of arachnoid cysts. Radiology. 1979 Mar;130(3):675-80.
11. Martinez-Lage JF, Perez-Espejo MA, Almagro MJ Lopez-Guerrero AL. Hydrocephalus and arachnoid cysts. Childs Nerv Syst. 2011 Oct;27(10):1643-52.

12. Martinez-Lage JF, Ruiz-Macia D, Valenti JA, Poza M. Development of a middle fossa arachnoid cyst. A theory on its pathogenesis. Childs Nerv Syst. 1999 Mar;15(2-3):94-7.

13. Schumacher M, Baust W, Terwey B. Unusual combination of cerebral dysplasias. Report of two cases. Neurochirurgia (Stuttg). 1986 Sep;29(5):210-4.

14. Zanini MA, Gabarra RC, Faleiros AT, Freitas CC, Alves A. [Cerebral aneurysm and arachnoid cyst: About a case with intracystic hemorrhage]. Arq Neuropsiquiatr. 2000 Jun;58(2A):330-5. Portuguese. 